\title{
Active Perception-Based Haptic Texture Sensor
}

\author{
Aiguo Song*, Yezhen Han, Haihua Hu, Lei Tian and Juan Wu \\ School of Instrument Science and Engineering, Southeast University, \\ Nanjing 210096, P. R. China
}

(Received May 21, 2012; accepted September 4, 2012)

Key words: texture sensor, haptic feedback robot, haptic display, active perception

Surface texture is one of the important cues for human beings to identify objects. Haptic texture measurement is necessary for object recognition by touch. This paper presents a novel design of a haptic texture sensor by imitating human active texture perception. A thin polyvinylidene fluoride (PVDF) film is used as the sensitive element to fabricate a high-accuracy, high-speed-response haptic texture sensor, and a mechanism is designed to produce the relative motion at a certain speed between the haptic texture sensor and the surface of the perceived object with constant contact force. Thus, the surface texture property can be measured as the output charge of the PVDF film of the sensor induced by the small height/depth variation of the moving object surface. The experiments reveal that the proposed active haptic sensor is effective in detecting the feature signals of surface texture, and the measurement signal can be used not only for the classification of the object surfaces, but also for haptic texture display in virtual reality.

\section{Introduction}

The sense of touch, one of the five senses of human beings, is known to be a critical sense for environment perception. The surface texture information is regarded to be an important cue to recognize objects. ${ }^{(1)}$ Therefore, in the fields of robotics, medicine, and virtual reality, being able to detect how smooth or rough a surface is, is an interesting feature of the sense of touch. For example, when a surgeon is performing laparoscopic surgery, whether the texture of tissues has changed is a noteworthy feature of organ lesion in the patient's body. The measurement of tissue texture of the organ being operated on by the surgeon is helpful in improving the quality of laparoscopic surgery. ${ }^{(2,3)}$ In a master-slave telerobot system, if the slave robot could detect the roughness of the touched object and the master device could accurately feed back the sense of touch to the operator, this would enable the system to be applicable to a wider variety of tasks.

${ }^{*}$ Corresponding author: e-mail: a.g.song@seu.edu.cn 
In virtual reality, building realistic haptic virtual environments requires the effective measurement of real-world surfaces. Texture measurement technology has also started to attract attention in the fields of food, fabrics, and cosmetics, ${ }^{(4)}$ e.g., Tanaka conducted texture evaluation of fabric and human skin using the output of a touch sensor. ${ }^{(5)}$

In surface metrology, textures are measured as roughness properties. The size of textual elements varies with the purpose of analysis. Compared with the properties of shape band geometry, texture comprises the spatial components with high spatial frequencies and small amplitudes, which can be further decomposed into components of higher frequencies and small amplitudes for analytical purposes. The patterns of general surface textures can be periodic, stochastic, or a combination of both.

Mukaibo et al. categorized tactile sensors into two types. ${ }^{(6)}$ One type is a grasping tactile sensor, which was created to control the grasping force of robot hands so as to not let an object slip out of the hand during its handling. The other type is the haptic texture sensor, which is used to sense the surface roughness of an object.

Although robot multi-axis force/torque sensors and grasping tactile sensors have been researched extensively, ${ }^{(7,8)}$ only a few studies have been conducted on the haptic texture sensors, because the effective measurement of texture is still a challenging task, and haptic texture sensors are relatively difficult to design. ${ }^{(9)}$ Yamada et al. developed a grasping tactile sensor by mimicking the structural features of human fingers to detect precise contact distribution and measure the surface softness of an object. ${ }^{(10)}$ Pai and Rizun developed a handheld wireless haptic texture sensor for haptic interaction in a virtual environment that can measure the magnitude of contact force between the probe tip of the sensor and solid surfaces. ${ }^{(11)}$ Mukaibo et al. designed a texture sensor by imitating the structure of human fingers. It is able to measure roughness, softness, and friction of the touched object.(6) Kim et al. developed a polymer-based microelectromechanical system (MEMS) tactile sensor array for surface texture measurement and classification. ${ }^{(12)}$ Hristu et al. created a deformable membrane tactile sensor adapting the deformable feature of human fingertips, ${ }^{(13)}$ and Ye et al. presented a systematic approach for sensing and reconstructing a periodic surface texture using a pen-type texture sensor. ${ }^{(14)}$

However, most of the existing methods make use of intrinsically passive texture sensors, which are only able to detect partial information of the surface such as touch, force, softness, and friction. Psychophysical research shows that the haptic texture perception of human fingers is an active process influenced by both the finger motion and applied force on the object surface. ${ }^{(15,16)}$ Lederman et al. investigated the variability of normal contact force applied on the surface by a finger or rigid probe when a human explores a surface texture, and demonstrated that actively applying force on the object surface with a periodic or repeated side-to-side motion greatly enhances the perception of texture. ${ }^{(17)}$

In this study, by mimicking the active texture perception process of a human, we develop a kind of haptic texture sensor for object surface texture measurement and identification. A thin polyvinylidene fluoride (PVDF) film is used as the sensitive element, because of its high piezoelectric effect and its unique physical properties, to fabricate a high-accuracy, high-speed-response haptic texture sensor, and a mechanism 
is designed to produce the relative motion at a certain speed between the haptic texture sensor and the surface of the perceived object with constant contact force.

The rest of the paper is organized as follows. Section 2 presents the idea of the overall design of the active haptic texture sensor system. Section 3 introduces the principle of the haptic texture sensor using a piezoelectric PVDF film, and $\S 4$ describes the haptic texture sensor mechanical design and circuit design method. The experiment results are discussed in $\S 5$. Finally, $\S 6$ concludes the paper.

\section{Overall Design of Active Haptic Texture Sensor System}

Texture perception of a human is a typical active process that usually consists of the following three steps. ${ }^{(18)}$ Step 1: the fingertip pushes the object surface with slight pressure and repeats a back-and-forth motion at speeds from 2 to $10 \mathrm{~cm} / \mathrm{s}$. The particles from the surface texture stimulate the touch receptors in skin tissues. Step 2: touch receptors convert the environment variation information into neural activity and send it to the brain cortex through the spinal cord, brainstem, and thalamus. Step 3: the cerebral cortex then resolves the perceived information, resulting in a specific texture feeling.

Smith et al. studied the texture surface exploration process of humans by the fingertip, and showed that the mean normal contact force exerted by a human being on the texture surface is $1.54 \pm 0.50 \mathrm{~N}$. ${ }^{(19)}$

The essence of human texture perception is that the touch receptors in skin tissue are sensitive to the dynamic touch force but insensitive to the static force. By imitating the above process of human active texture perception, we design a novel active sensor system of haptic texture using a piezoelectric PVDF film, which is sensitive to the dynamic force as well.

Figure 1 shows the schematic diagram of the proposed active haptic texture sensor system based on the PVDF film. It consists of a support frame, haptic texture sensor, contact force adjustment mechanism with a thin spring steel beam, DC motor with encoder, rotation disk connected with the motor shaft, and circuits. In this measurement system, the fingertip-type haptic texture sensor, consisting of a PVDF film and a force sensor, is installed on the bottom end of a shaft and perpendicular to the surface of the perceived object put on the rotation disk. The shaft connects the haptic texture sensor to the thin spring steel beam. Before measuring the object surface texture, the adjustment nut in the contact force adjustment mechanism is turned to make the thin spring steel beam bend so as to keep the haptic texture sensor in contact with the object surface at a certain normal contact force. Then, the motor is controlled, by a computer through the drive circuit, to rotate at a certain constant speed, so that the texture sensor slides along the surface of the object laid on the rotation disk. The surface texture property can be obtained by measuring the output charge of the PVDF film of the sensor, which is induced by the small height/depth variation of the moving object surface.

A photograph of the active haptic texture sensor system is shown in Fig. 2. The support frame, contact force adjustment mechanism, shaft, and rotation disk are made of aluminum. The support frame is $160 \mathrm{~mm}$ high with three cylindrical pillars and a round plate of $70 \mathrm{~mm}$ diameter and $4 \mathrm{~mm}$ thickness. The DC motor is installed on the round 


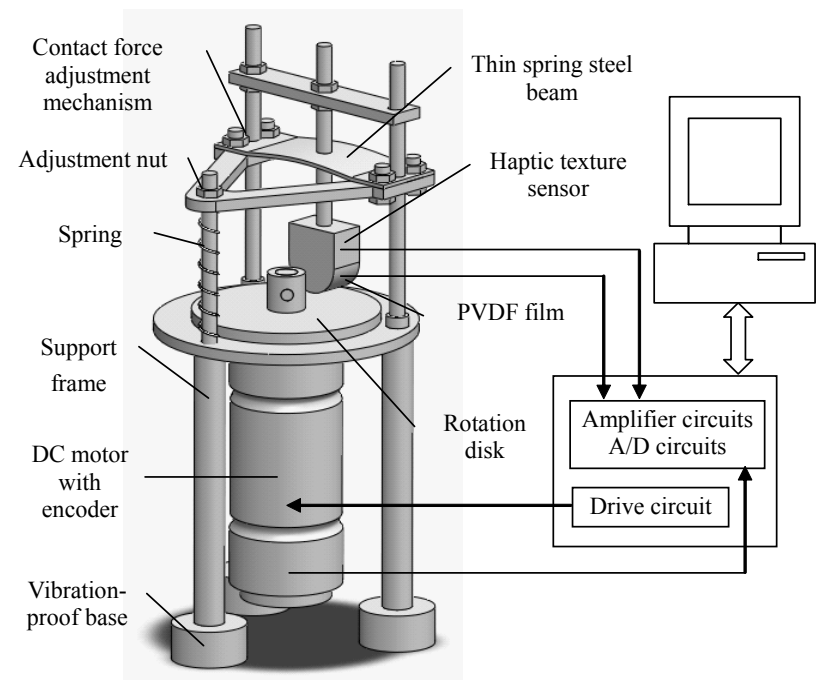

Fig. 1. Structure of active haptic texture sensor system.

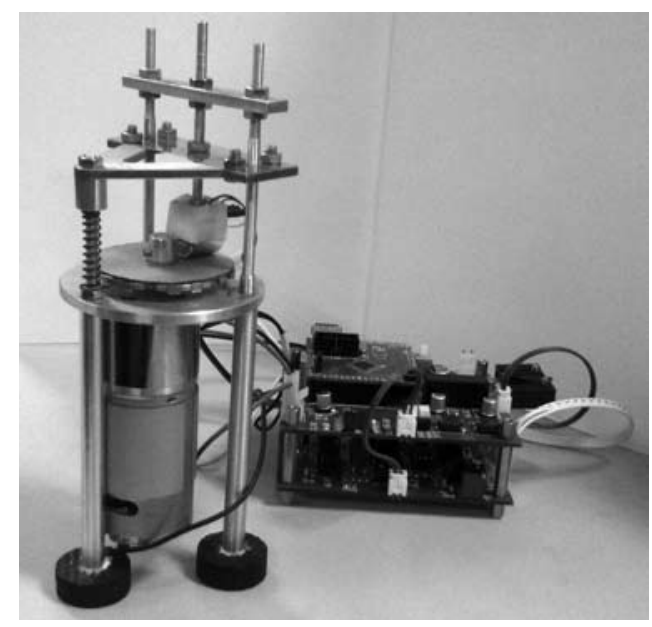

Fig. 2. Photograph of the active haptic texture sensor system.

plate. The rotation disk is $2 \mathrm{~mm}$ thick with a $50 \mathrm{~mm}$ diameter. The shaft is $40 \mathrm{~mm}$ long with $4 \mathrm{~mm}$ diameter, and its bottom end is designed as a small plate of $17 \mathrm{~mm}$ diameter and $2.5 \mathrm{~mm}$ thickness and used for haptic texture sensor installation. The detailed design of the haptic texture sensor is described in the following sections. 


\section{Principle of Texture Measurement Using PVDF Film}

PVDF is a semicrystalline polymer of approximately $50-65 \%$ crystallinity. The polymer consists of long-chain molecules with the repeated unit $\mathrm{CF}_{2}-\mathrm{CH}_{2}$. The reason for the strong piezo-pyroelectric activity is related to the large electronegativity of fluoride atoms in comparison with the carbon atoms, and thus accommodates a large dipole moment. Furthermore, PVDF is flexible, light, tenacious, and inexpensive compared with other piezoelectric materials. Thus, a PVDF film is highly suitable for tactile sensor fabrication. ${ }^{(20)}$

Figure 3 is a schematic of a PVDF film. The piezoelectric coefficient matrix for this form is usually expressed as ${ }^{(21)}$

$$
d_{i j}=\left[\begin{array}{cccccc}
0 & 0 & 0 & 0 & d_{15} & 0 \\
0 & 0 & 0 & d_{24} & 0 & 0 \\
d_{31} & d_{32} & d_{33} & 0 & 0 & 0
\end{array}\right]
$$

The axes used are defined in terms of the drawn direction (direction 1), normal to the drawn direction in the plane of the film (direction 2), and normal to the plane of the film (direction 3), as shown in Fig. 3.

The sensitivity of the PVDF film is dependent on the direction of measurement, that is, drawn, transverse, or thickness. When a tensile force is applied in direction 1 (drawn direction), the output charge is expressed as

$$
\frac{Q}{A_{3}}=d_{31} \frac{F}{A_{1}}=d_{31} \sigma_{1}
$$

Similarly, the output charge due to stress in direction 2 (transverse direction) is expressed as

$$
\frac{Q}{A_{3}}=d_{32} \frac{F}{A_{2}}=d_{32} \sigma_{2}
$$

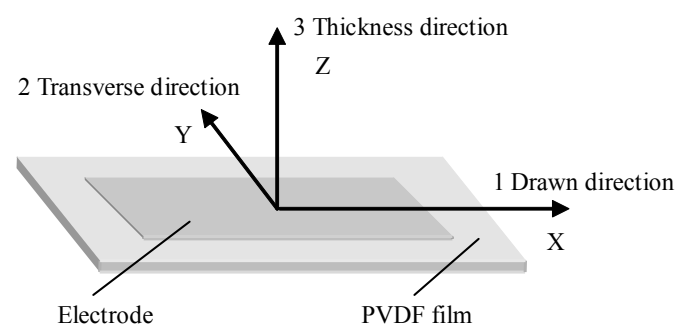

Fig. 3. Schematic of PVDF film showing the conventional identification of the axes. 
where $Q$ is the output charge, $A_{3}$ is the electroded area of the PVDF film, $A_{1}$ and $A_{2}$ are the cross-sectional areas of the film perpendicular to the direction of the applied force, $d_{31}$ is the piezoelectric strain coefficient in the drawn direction, $d_{32}$ is the piezoelectric strain coefficient in the transverse direction, $F$ is the applied force, and $\sigma_{1}$ and $\sigma_{2}$ are the applied tensile stress in the drawn direction and the transverse direction, respectively.

When a PVDF film is compressed by a probe on a rigid flat surface, assuming that both the flat surface and the probe are friction-free, the film is free to expand laterally and the output charge can be expressed as

$$
\frac{Q}{A_{3}}=d_{33} \frac{F}{A_{3}}=d_{33} \sigma_{3},
$$

where $d_{33}$ is the piezoelectric strain coefficient in direction 3 .

Normally, in tactile sensing, the output charge of the PVDF film is due to a combination of $d_{31}, d_{32}$, and $d_{33}$. For a given applied force, the output charge from the film in the lateral direction is much higher than that in the thickness direction. This is because of the extreme thinness of the PVDF film, which results in much higher stresses being applied to the film. For the commercially available PVDF film, $-d_{33} \geq d_{31}>d_{32}>0$, and the value of $d_{31}$ is normally one order higher than that of $d_{32}$. Thus, as the sensitive element for a texture sensor, eq. (1) can be rewritten as

$$
d_{i j}=\left[\begin{array}{cccccc}
0 & 0 & 0 & 0 & 0 & 0 \\
0 & 0 & 0 & 0 & 0 & 0 \\
d_{31} & 0 & d_{33} & 0 & 0 & 0
\end{array}\right]
$$

The principle of the haptic texture sensor using the piezoelectric PVDF film is that the PVDF film touches the object surface under a constant normal force and slides on the surface. During the sliding movement, a micro-unit of the PVDF film will be compressed by the fine particles on the surface, resulting in an amount of charge. Measuring the output charges of the PVDF film yields the surface texture characteristics.

The PVDF texture sensor model is shown in Fig. 4. Usually, the electrode fully covers the surface of the PVDF film, that is, the electroded area $A_{3}$ equals the surface area of PVDF film $\Omega$.

Let $\sigma(x, y, t)$ denote the strain of point $(x, y, t)$ at time $t$ on the surface of the PVDF film. Then, the output charge of the PVDF texture sensor caused by the strain change can be expressed as

$$
Q(t)=Q\left(t_{0}\right)+\oiint_{\Omega}\left[\int_{0}^{t} d_{31} \frac{\partial \sigma_{1}(x, y, t)}{\partial t}+d_{33} \frac{\partial \sigma_{3}(x, y, t)}{\partial t}\right] d x d y
$$

where $Q\left(t_{0}\right)$ is the output charge of the PVDF texture sensor at the initial time $t_{0}$.

Because the voltage between the upper electrode and bottom electrode of the PVDF film is equal to the voltage of the amplifier equivalent resistance, the leakage current of the texture sensor is 


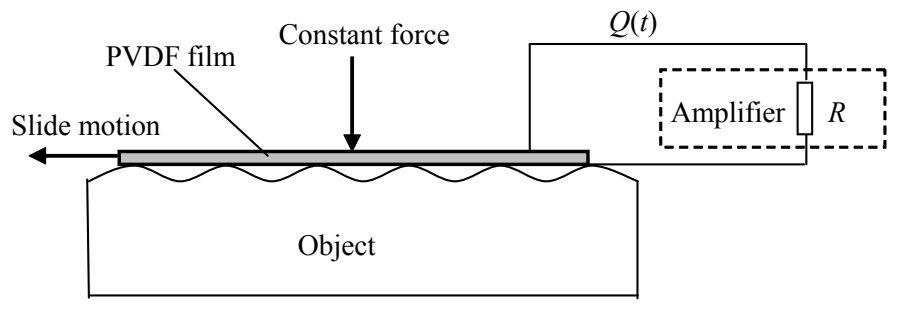

Fig. 4. Model of the PVDF texture sensor.

$$
i \approx \frac{Q(t)}{R \cdot C}
$$

where $C$ is the equivalent capacitance of the PVDF piezoelectric film.

When the PVDF texture sensor is connected to the amplifier circuits, the leakage current should be considered, thus

$$
\begin{gathered}
Q(t)=Q\left(t_{0}\right)+\oiint_{\Omega}\left[\int_{0}^{t} d_{31} \frac{\partial \sigma_{1}(x, y, t)}{\partial t}+d_{33} \frac{\partial \sigma_{3}(x, y, t)}{\partial t}\right] d x d y-\int_{0}^{t} i d t \\
Q(t)=Q\left(t_{0}\right)+\oiint_{\Omega}\left[\int_{0}^{t} d_{31} \frac{\partial \sigma_{1}(x, y, t)}{\partial t}+d_{33} \frac{\partial \sigma_{3}(x, y, t)}{\partial t}\right] d x d y-\int_{0}^{t} \frac{Q(t)}{R C} d t .
\end{gathered}
$$

Because the electrode film to which the PVDF film is glued is usually rigid in the horizontal direction, $\sigma_{1}=\sigma_{2}=0$. Assuming the strain is uniform on the sensitive area of the PVDF film and that the initial output charge $Q\left(t_{0}\right)=0$, then the total electric charge $Q(t)$ induced at each surface electrode of the PVDF film by the strain at the contact area can be given as

$$
Q(t)=\Omega \cdot d_{33} \int_{0}^{t} \frac{\partial \sigma_{3}(x, y, t)}{\partial t} d t-\int_{0}^{t} \frac{Q(t)}{R C} d t
$$

When strain $\sigma_{3}$ is changed as a step function, the solution of eq. (10) is

$$
Q(t)=\Omega \cdot d_{33} \cdot e^{-\frac{t}{R C}} \cdot U(t)=Q_{\Omega} e^{-\frac{t}{T}} U(t),
$$

where $U(t)$ is the step function, $T=R C$ is the response time constant, and $Q_{\Omega}=\Omega \cdot d_{33}$ is the amplitude of output charge caused by strain $\sigma_{3}$ in the electrode area.

Equation (11) illustrates that the output charge of the PVDF texture sensor will 
exponentially decrease with time variable $t$ owing to the leakage current of the sensor and the amplifier. This implies that the haptic texture sensor using the PVDF film as the sensing element is unable to measure the static force.

\section{Active Haptic Texture Sensor Design}

\subsection{Mechanical design of haptic texture sensor based on PVDF film}

The mechanical structure of the haptic texture sensor is shown in Fig. 5(a). It is designed as a fingertip-type shape. The haptic texture sensor comprises the electroded PVDF film, infilling, the force sensor, and the base. The base is a cylinder connected to the haptic texture sensor at the bottom end of the shaft. The silicon rubber is used as the infilling to fill in the cavity formed by the base, force sensor, and electroded PVDF film, and is capable of transferring the contact force from the PVDF film to the force sensor owing to its human-tissue-like softness property. A latex membrane covers the hemispherical surface of the silicon rubber as a protective layer. Then, the electroded PVDF film is glued to the latex membrane surface. Figure 5(b) shows a photograph of the haptic texture sensor.

The function of the electroded PVDF film is to measure the strain variation corresponding to the surface texture change when a relative sliding motion occurs between the haptic texture sensor and the surface of the object. Figure 6 is a photograph of the electroded PVDF film of $10 \mathrm{~mm}$ width and $30 \mathrm{~mm}$ length. Two metal electrodes fully cover each surface of the piezoelectric PVDF film with a $2 \times 6 \mathrm{~mm}^{2}$ electrode pin.

During the process of the active measurement of the surface texture, we should wring the screw nut so as to bring the haptic texture sensor into contact with the object surface under a constant normal force. Here, we use the FSG1500g touch force sensor manufactured by Honeywell Corporation, shown in Fig. 7, to measure the normal contact

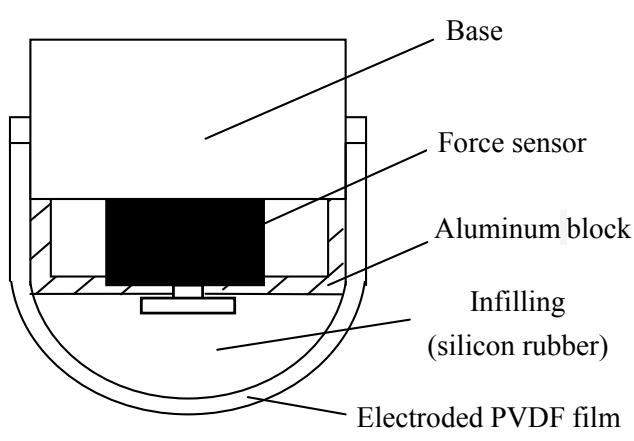

(a)

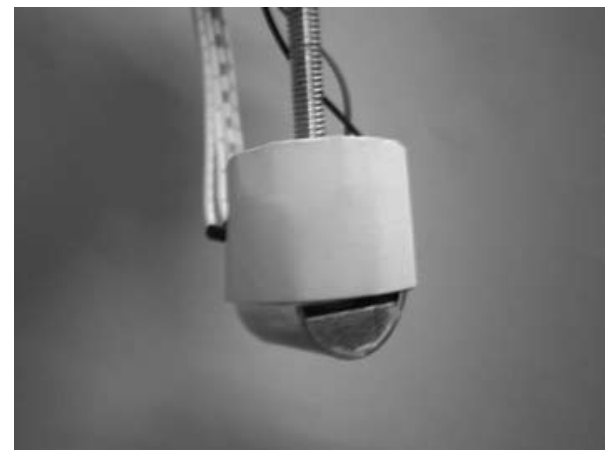

(b)

Fig. 5. PVDF-based haptic texture sensor: (a) structure of haptic texture sensor and (b) photograph of haptic texture sensor. 

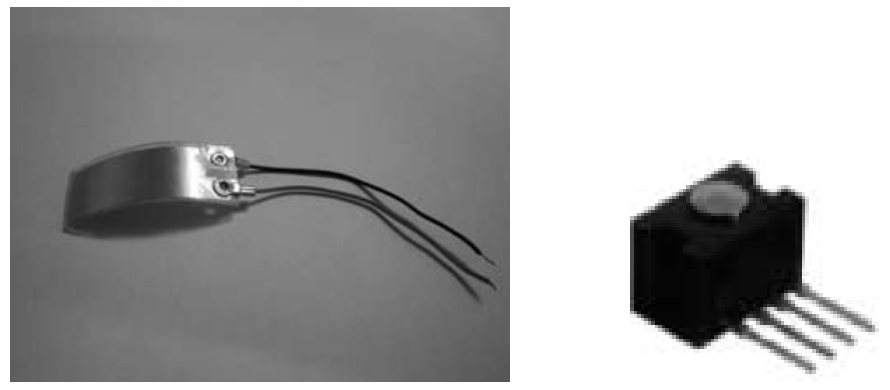

Fig. 6 (left). Electroded PVDF film.

Fig. 7 (right). FSG1500g touch force sensor.

force between the PDVF film and the object surface. The touch force sensor FSG $1500 \mathrm{~g}$ is installed on the bottom of the cylindrical base. It has low power consumption owing to its working principle of silicon piezoresistance. Its measurement range is $15 \mathrm{~N}$ with a measurement precision of $0.5 \%$ F.S., and its resistance to overload reaches $55 \mathrm{~N}$.

\subsection{Circuit design}

The circuit architecture of the haptic texture sensor is shown in Fig. 8. It consists of a conditioning circuit for the PVDF film output charge signal, a conditioning circuit for the force sensor output voltage signal, a drive circuit for DC motor control, and an I/O card connected to the computer bus.

\subsubsection{Conditioning circuit design for PVDF film output}

To convert the electrical charge generated by PVDF into voltage signals, a suitable conditioning circuit must be designed. To achieve this, some requirements should be taken into account. a) PVDF films generate electric charges when suffering from force deformation, so the charge amplifier should be used in the first stage of the circuit. b) As the haptic texture signal may be disrupted by power-line-related frequency noise and high-frequency noise, some filters should be used to eliminate such noise. c) In the conditioning circuit, the texture signal must be amplified while the noise should simultaneously be reduced. Therefore, the noise-reducing circuit and amplification circuit is particularly important.

To meet the three requirements, the structure of the conditioning circuit is designed and shown in Fig. 9. An LF356 operational amplifier, which has high input impedance and small power consumption and is suitable as a weak signal preamplifier, is chosen as the charge amplifier to convert the electric charges into a voltage signal. Then, the amplitude of the voltage signal is doubled through an amplifier before the first notch filter is used to reduce the $50 \mathrm{~Hz}$ power-line interference. After that, the amplitude of the signal is amplified five times. Since the power-line interference is amplified at the same time, the second notch filter is used to further remove the power-line interference 


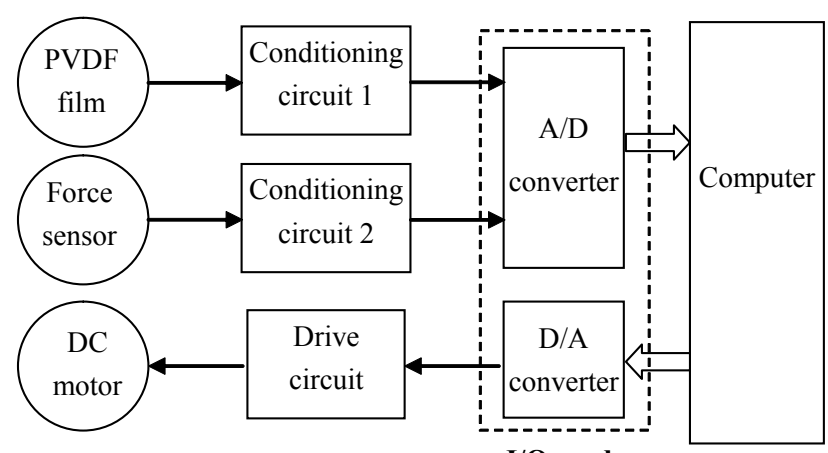

I/O card

Fig. 8. Circuit architecture of the haptic texture sensor.

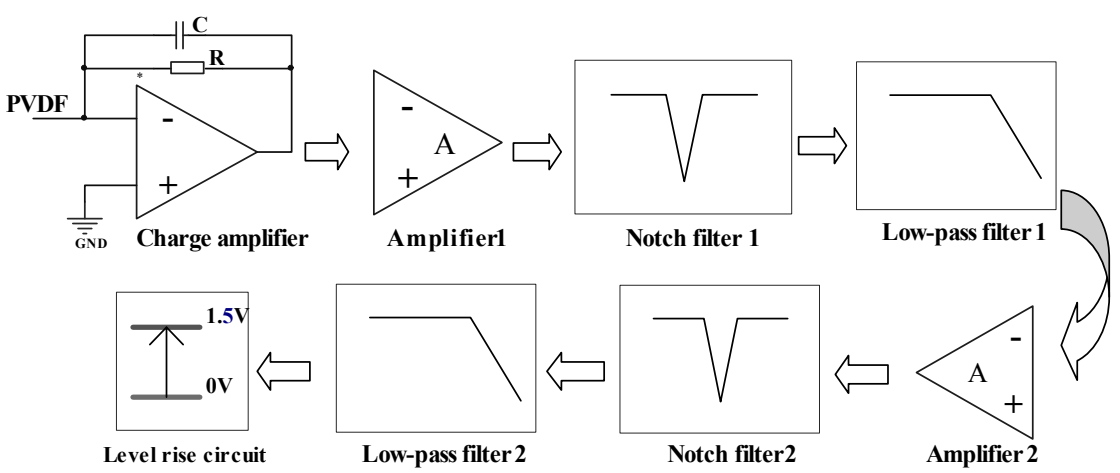

Fig. 9. Conditioning circuit structure.

and reduce the noise level to a very small value. Subsequently, there are two low-pass filters in this circuit. The first one, between notch filter 1 and amplifier 2 with voltage gain set as 4, is used to eliminate high-frequency noise from the source. The second one, between notch filter 2 and level rise circuit, is set to reduce the high-frequency noise amplified by amplifier 2 .

Note that the two amplifiers and two notch filters used in this design ensure that the weak texture signal can be amplified and measured properly while simultaneously reducing the power-line interference as much as possible.

\subsubsection{Conditioning circuit for touch force sensor}

The conditioning circuit for touch force sensor FSG1500g consists of a Wheatstone full-bridge circuit in the force sensor, differential amplifier, and signal amplifier, as shown in Fig. 10. The differential amplifier is set as the first amplifier in order to reject 


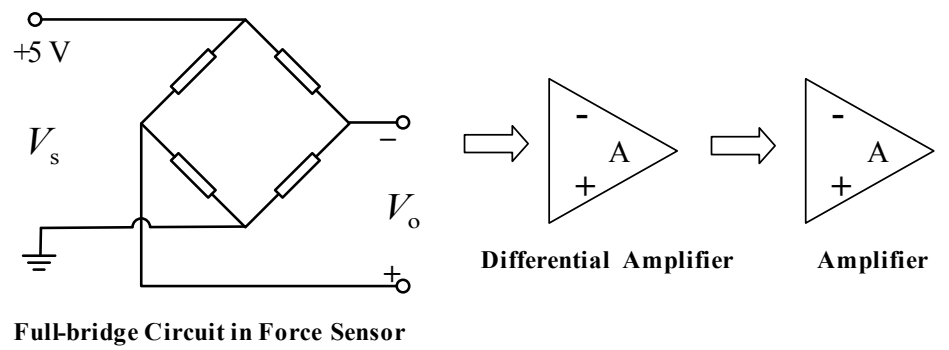

Fig. 10. Conditioning circuit for touch force sensor FSG1500g.

common mode error. Two classes of amplification consisting of a differential amplifier and an amplifier can also realize an impedance match no mater how the impedance of the Wheatstone full-bridge circuit changes.

\subsubsection{Drive circuit for DC motor control}

The H-bridge circuit is used to drive the DC motor. The microcomputer unit C8051 sends PWM signals of a certain duty ratio through the optical coupler isolation unit to the H-bridge circuit for DC motor control, as seen in Fig. 11. We change the duty ratio of the PWM signal so as to control the speed of the motor running within the range of human finger moving speed during active touching of the surface texture. Here, Freescale's MC33887 chip is selected as the H-bridge driver chip, which has a wide range of operating voltage of 5-28 V, output current up to $5 \mathrm{~A}$, and current protection and current feedback functions.

\section{Experimental Results}

We choose samples of smooth calendar paper, woolen fabric, and kraft paper, as seen in Fig. 12, as three types of perceived objects for the surface texture sensing experiment. During the experiment, in order to imitate human active texture perception, the rotation speed of the motor is set to be $1 \mathrm{round} / \mathrm{s}$, which is within the fingertip speed range of 2 $-10 \mathrm{~cm} / \mathrm{s},{ }^{(18)}$ and the pressing force of the haptic texture sensor applied on the perceived object surface is set to be $1.5 \mathrm{~N}$, which is within the fingertip touch force range of $1.54 \pm 0.50$ $\mathrm{N} .{ }^{(19)}$ The texture signals of the above three objects are shown in Fig. 13.

It is obvious in Fig. 13 that the signal amplitude of the smooth calendar paper is significantly less than those of the other two objects, indicating that its surface texture has more delicate particles and feels almost smooth. There are several periodic and regular spikes in the results for the woolen fabric, and texture features are very clear, indicating that the surface texture of the woolen fabric is very regular. The signal amplitude for the kraft paper is relatively high, and the signal has more irregular spikes, indicating that the surface texture feels rougher than the first two. 


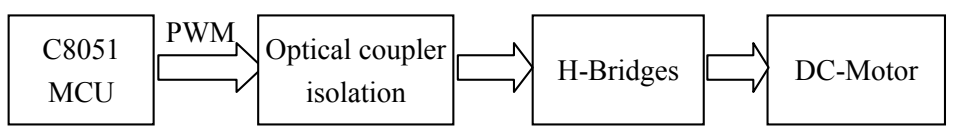

Fig. 11. Drive circuit for DC motor control.

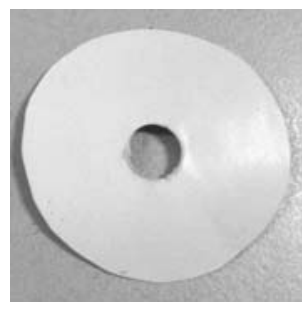

(a)

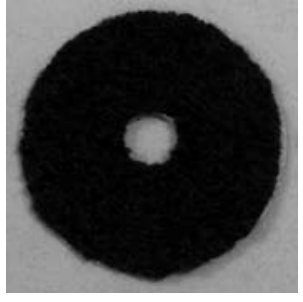

(b)

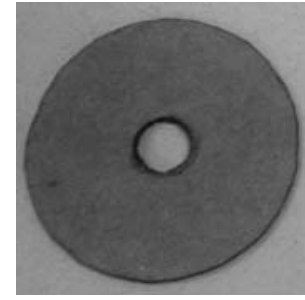

(c)

Fig. 12. Three types of perceived objects: (a) smooth calendar paper, (b) woolen fabric, and (c) kraft paper.

The power spectrum density of the above three texture signals is shown in Fig. 14. Each peak in the power spectral density map represents a kind of texture feature in the frequency domain, and the height of the peak reflects the energy amplitude of the corresponding texture characteristics.

It can be seen that the texture characteristics of the three samples are concentrated in the range of 1-20 Hz. The smooth calendar paper peaks are small with less energy, indicating a single texture feature. Two obvious peaks at 3 and $7 \mathrm{~Hz}$ for woolen fabric mean that its texture feature can be characterized by two main frequencies. The power spectrum of the kraft paper has numerous high peaks.

Therefore, the texture characteristics in the frequency domain are suitable for object classification, while the measurement signal of the texture property in the time domain is suitable for haptic texture display in virtual reality and teleoperation.

\section{Conclusions}

We developed an active haptic texture sensor system for surface texture measurement by imitating the human active texture perception process. A thin PVDF film was used as the sensitive element to fabricate the haptic texture sensor, and a mechanism was designed to produce the relative motion at a certain speed between the haptic texture sensor and surface of the perceived object with constant contact force. The experiments 
(a)

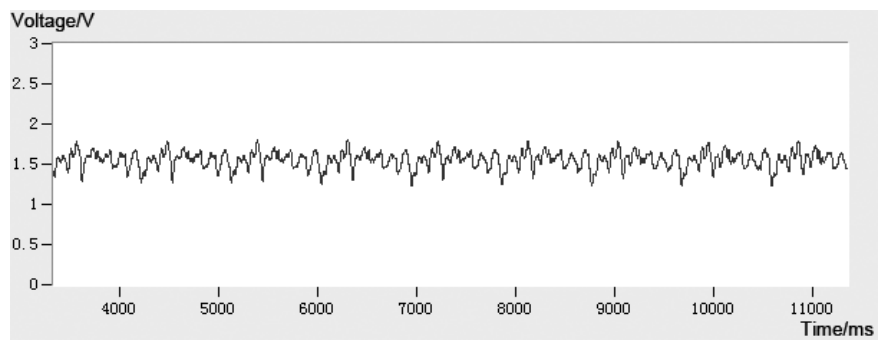

(b)

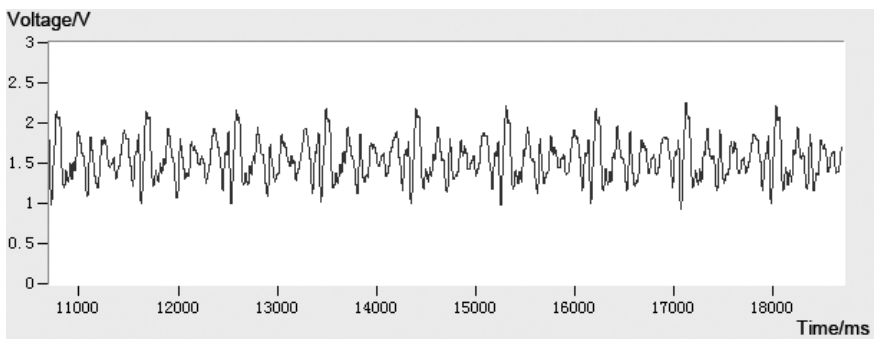

(c)

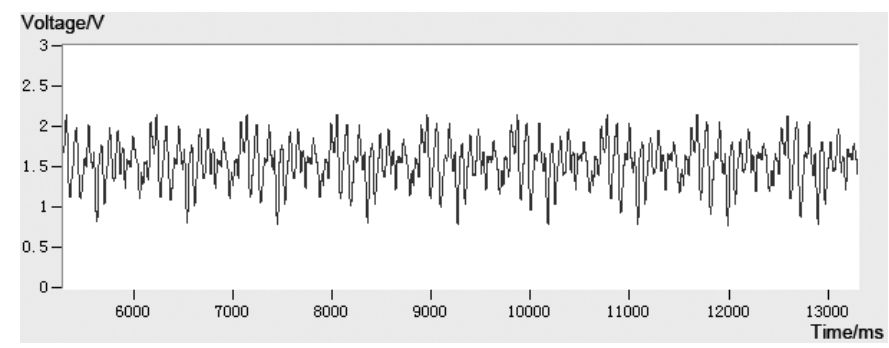

Fig. 13. Texture signals of the three objects: (a) smooth calendar paper, (b) woolen fabric, and (c) kraft paper.

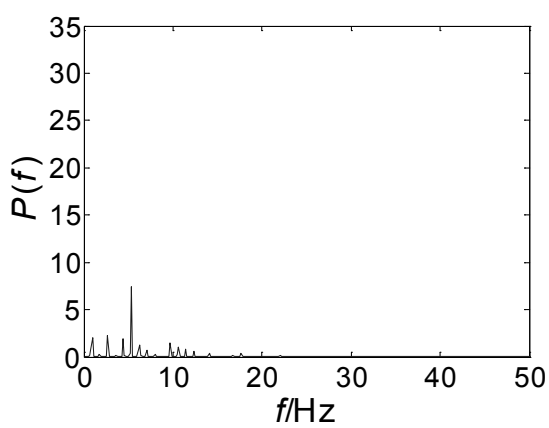

(a)

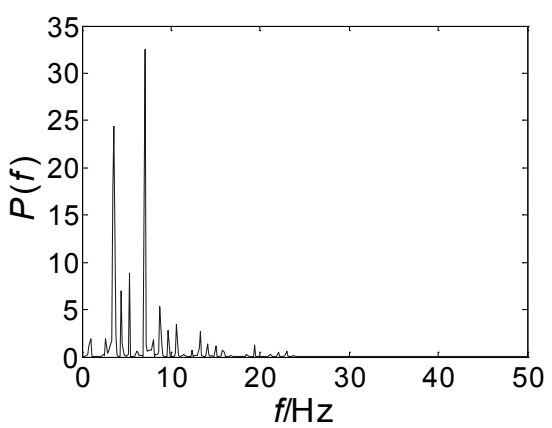

(b)

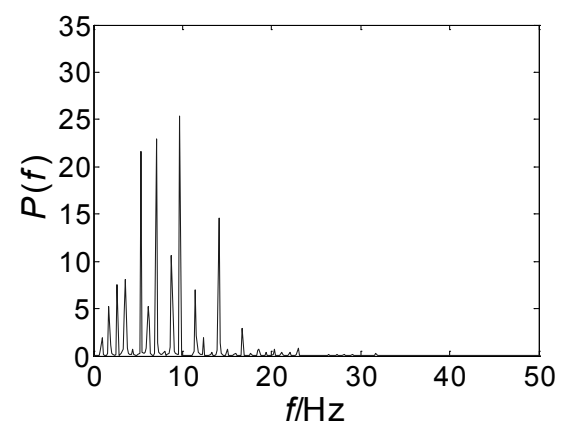

(c)

Fig. 14. Texture signal power spectrum densities of the three objects: (a) smooth calendar paper, (b) woolen fabric, and (c) kraft paper. 
revealed that the proposed active haptic sensor is an effective device for detecting the feature signals of surface texture, and the measurement signal can be used not only for object recognition, but also for haptic texture display in virtual reality and teleoperation.

\section{Acknowledgements}

This study was supported by the Natural Science Foundation of China under grant numbers 61075068, 60905045, 61105075, and 61272379, Natural Science Foundation of Jiangsu Province under grant number BK2010063, and Science and Industrial Research Project of Changzhou City under grant number CE20100022.

\section{References}

1 M. Takasaki, T. Nara and T. Mizuno: IEEE Int. Symp. on Industrial Electronics 2003 (IEEE, Rio de Janeiro, 2003) p. 342.

2 B. C. C. Khoo, M. P. C. McQueen and W. J. Sandle: J. Biomed. Eng. 13 (1991) 489.

3 V. Hayward, O. R. Astley, M. Cruz-Hernandez, D. Grant and G. Robles-De-La-Torre: Sens. Rev. 24 (2004) 16.

4 M. Taniwaki, N. Sakurai and H. Kato: Food Res. Int. 43 (2010) 814.

5 M. Tanaka: J. Mater. Process. Technol. 108 (2001) 253.

6 Y. Mukaibo, H. Shirado, M. Konyo and T. Maeno: Int. Conf. on Robotics and Automation 2005 (IEEE, Barcelona, 2005) p. 2576.

7 A. Bicchi: Rob. Autom. Syst. 10 (1992) 269.

8 A. Song, J. Wu, G. Qin and W. Huang: Measurement 40 (2007) 883.

9 M. H. Lee: Int. J. Rob. Res. 19 (2000) 636.

10 D. Yamada, T. Maeno and Y. Yamada: J. Rob. Mechatron. 14 (2002) 140.

11 D. K. Pai and P. R. Rizun: 11th Symp. on Haptic Interfaces for Virtual Environment and Teleoperator Systems 2003 (IEEE, Los Angeles, 2003) p. 3.

12 S. H. Kim, J. Engel, C. Liu and D. L. Jones: J. Micromech. Microeng. 15 (2005) 912.

13 D. Hristu, N. Ferrier and R. W. Brockett: Int. Conf. on Robotics and Automation 2000 (IEEE, San Francisco, 2000) p. 508.

14 X. Ye, H. Moon and H. R. Choi: Intell. Serv. Rob. 3 (2010) 183.

15 K. Drewing and M. O. Ernst: Brain Res. 1078 (2006) 92.

16 K. Drewing and L. Kaim: Atten. Percep. Psychophys. 71 (2009) 1174.

17 S. J. Lederman, R. D. Howe, R. L. Klatzky and C. Hamilton: 12th Int. Symp. on Interfaces for Virtual Environment and Teleoperator Systems 2004 (IEEE, Chicago, 2004) p. 154.

18 J. Pasquero and V. Hayword: EUROHAPTICS 2003 (Eurohaptics, Dublin, 2003) p. 94.

19 A. M. Smith, G. Basile, J. Theriault-Groom, P. Fortier-Poisson, G. Campion and V. Hayward: Exp. Brain Res. 202 (2010) 33.

20 M. A. Qasaimeh, S. Sokhanvar, J. Dargahi and M. Kahrizi: J. Microelectromech. Syst. 18 (2009) 195.

21 J. Dargahi: Sens. Actuators, A 80 (2000) 23. 


\begin{abstract}
About the Authors
Aiguo Song earned his B.S. degree in Automatic Control in 1990 and M.S. degree in Measurement and Control in 1993 from Nanjing Aeronautics and Astronautics University, Nanjing, P. R. China, and Ph.D. degree in Measurement and Control from Southeast University, Nanjing, P. R. China in 1996. He is currently a Professor with the School of Instrument Science and Engineering, Southeast University, P. R. China. His research interests are on teleoperation, haptic display, robot haptic sensors, and rehabilitation robot. He is a member of the Chinese Instrument and Control Association and Chinese Robot Association and a senior member of IEEE.
\end{abstract}

Yezhen Han earned her B.S. degree in Measurement and Control from Southeast University, Nanjing, P. R. China in 2012, where she is currently working toward her M.S. degree in Measurement and Control. Her research interests include haptic texture sensor and haptic display.

Haihua Hu earned his B.S. degree in Measurement and Control from Southeast University, Nanjing, P. R. China in 2012, where he is currently working toward his M.S. degree in Measurement and Control. His research interests include robot sensor and control.

Lei Tian earned her B.S. degree in Electronic and Information Engineering in 2003 and M.S. degree in Measurement and Control in 2007 from Jilin University, Changchun, P. R. China. She is currently working toward her Ph.D. degree in Measurement and Control at Southeast University, Nanjing, P. R. China. Her research interests include haptic texture sensor and haptic display.

Juan Wu earned her B.S., M.S., and Ph.D. degrees in Measurement and Control from Southeast University, Nanjing, P. R. China, in 1999, 2002, and 2005, respectively. She is currently an associate Professor with the School of Instrument Science and Engineering, Southeast University, P. R. China. Her research interests include haptic display, hapticbased telerobot, and robot sensor. 